OPEN ACCESS

Edited by:

Luis C. Anton,

Consejo Superior de Investigaciones Cientificas (CSIC), Spain

Reviewed by:

Ludger Klein,

Ludwig Maximilian University of

Munich, Germany

Heung Kyu Lee,

Korea Advanced Institute of Science

and Technology, South Korea

Payel Sil,

National Institute of Environmental

Health Sciences (NIEHS),

United States

*Correspondence:

Christian Münz

christian.muenz@uzh.ch

Specialty section:

This article was submitted to Antigen Presenting Cell Biology,

a section of the journal

Frontiers in Immunology

Received: 11 November 2020

Accepted: 18 January 2021

Published: 25 February 2021

Citation:

Münz C (2021) The Macroautophagy Machinery in $\mathrm{MHC}$

Restricted Antigen Presentation.

Front. Immunol. 12:628429.

doi: 10.3389/fimmu.2021.628429

\section{The Macroautophagy Machinery in MHC Restricted Antigen Presentation}

\author{
Christian Münz \\ Viral Immunobiology, Institute of Experimental Immunology, University of Zürich, Zürich, Switzerland
}

Autophagy-related (ATG) gene products regulate macroautophagy, LC3-associated phagocytosis (LAP) and LC3-dependent extracellular vesicle loading and secretion (LDELS). These processes also influence antigen processing for presentation on major histocompatibility complex ( $\mathrm{MHC}$ ) molecules to $\mathrm{T}$ cells. Here, I summarize how these different pathways use the macroautophagy machinery, contribute to MHC class I and II restricted antigen presentation and influence autoimmunity, tumor immunology and immune control of infectious diseases. Targeting these different pathways should allow the regulation of intracellular and extracellular antigen presentation to T cells to modulate protective and pathological immune responses.

Keywords: cytotoxic CD8+ $\mathrm{T}$ cells, helper CD4+ $\mathrm{T}$ cells, cross-presentation, LC3-associated phagocytosis, exocytosis

\section{THE MACROAUTOPHAGY MACHINERY}

Yoshinori Ohsumi described in a landmark paper in 199315 genes that are required in yeast to survive starvation (1). These formed the core of the more than 40 autophagy-related proteins that regulate macroautophagy, one of at least three pathways by which cytoplasmic constituents are imported into lysosomes for degradation (2). This machinery consists of a protein kinase complex, a lipid kinase complex, enzymes that couple ubiquitin-like molecules to membranes and recruit substrates to them, as well as a fusion machinery that delivers the result of the first three complexes, a double-membrane surrounded autophagosome, to lysosomes for the degradation of the cargo and the inner membrane of autophagosomes (Figure 1). Many of the molecular components of this machinery are abbreviated as ATG (autophagy-related) proteins. The protein kinase complex of ATG1/ULK1 gets inhibited by mammalian target of rapamycin complex 1 (mTORC1) and activated by AMP kinase (AMPK) via differential phosphorylation. This allows eukaryotic cells to initiate macroautophagy upon nutrient deprivation. The ATG1/ULK1 complex then phosphorylates itself and components of all stages of autophagosome maturation and degradation (3). However, one of its main targets is the VPS34 phosphatidylinositol 3 (PI3) kinase complex containing ATG6/ Beclin-1. This complex generates the phospholipid PI3P that recruits the ATG8/LC3B lipidation machinery to membranes via WIPI proteins, predominantly WIPI2. The ATG8/LC3B lipidation machinery consists of the E1-like enzyme ATG7. The E2-like enzymes ATG3 and 10, and the E3-like enzyme ATG5-ATG12-ATG16L1 that finally couples the six mammalian ATG8 orthologues LC3A, LC3B, LC3C, GABARAP, GABARAP-L1, and GABARAP-L2 primarily to phosphatidylethanolamine (PE) in the forming autophagosome that is called isolation membrane or phagophore. Attached to the phagophore membrane these ATG8 orthologues assist in membrane fusion during phagophore extension by ATG9 containing vesicles and ATG2 mediated lipid transfer 


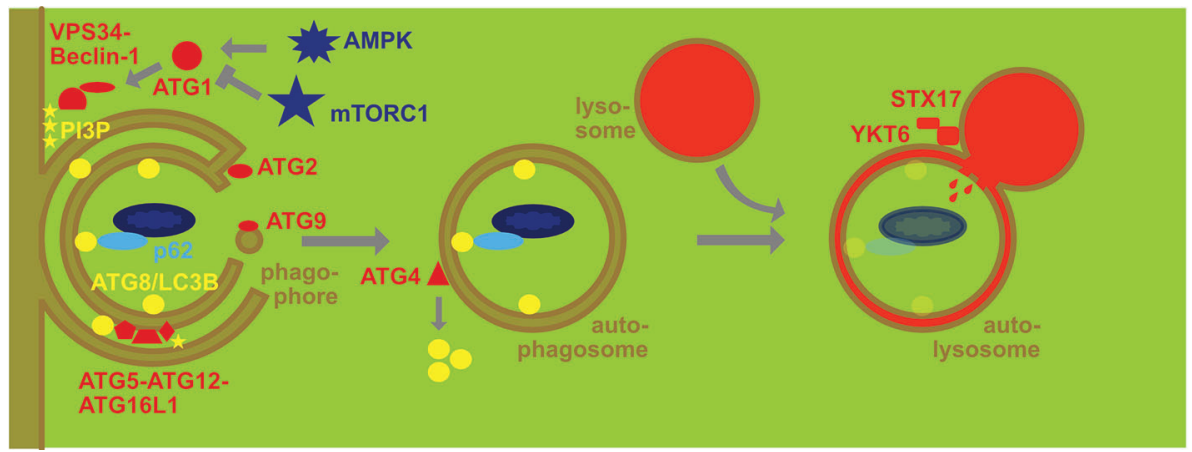

FIGURE 1 | Molecular machinery of macroautophagy. The protein kinase ATG1/ULK1 is activated by AMP kinase (AMPK) and inhibited by mammalian target of rapamycin complex 1 (mTORC1). It then activates the lipid kinase complex containing VPS34 and Beclin-1 to generate phosphatidylinositol-3-phosphate (PI3P) that then recruits the ATG8/LC3B lipidation complex ATG5-ATG12-ATG16L1 that couples ATG8/LC3B to phosphatidylethanolamine at the inner and outer membrane of the phagophore. Membranes are donated to the phagophore via ATG2 associated channels and ATG9 carrying vesicles. Once this double membrane closes around cargo that is recruited by macroautophagy receptors like p62 via binding to ATG8/LC3 to form an autophagosome ATG4 recycles ATG8/LC3B from the outer membrane. The autophagosome fuses then with late endosomes and lysosomes in a syntaxin 17 (STX17) and YKT6 dependent fashion for degradation of the cargo and the inner autophagosome membrane with coupled ATG8/LC3B.

(4-6). Prior to their lipidation the ATG8 orthologues need to be processed by the ATG4 proteases (ATG4A-D in higher eukaryotes) that also remove them from the outer membrane upon autophagosomes completion (7). The ATG8 orthologues also serve as anchors to recruit macroautophagy cargo to phagophores. Proteins with LC3-interacting regions (LIRs) bind to ATG8 orthologues and then the phagophore grows around the respective cargo, including damaged mitochondria, chloroplasts, ribosomes, proteasomes, endoplasmic reticulum, protein aggregates, damaged endosomes, bacteria, and some viral capsids. These LIR containing autophagy receptors, like sequestosome $1 /$ p62 or NBR1 that cross-link for example ubiquitinated cytoplasmic constituents with LC3B (8). After removal of most lipidated ATG8 orthologues from the outer membrane of the completed autophagosome, residual autophagy receptor binding at this site supports autophagosome transport along microtubules and recruitment of the fusion machinery with lysosomes or late endosomes $(9,10)$. Finally, the soluble $\mathrm{N}$-ethylmaleimide-sensitive-factor attachment receptors (SNAREs) syntaxin 17 and YKT6 are required for autophagosome fusion with lysosomes $(11,12)$. In the resulting autolysosome macroautophagy substrates and the inner autophagosomal membrane are degraded by lysosomal hydrolases. Therefore, macroautophagic flux is coupled to lysosomal activity and transcriptionally linked to transcription factor EB (TFEB) the master transcription factor of lysosomal biogenesis (13). The resulting molecular building blocks of lysosomal degradation, including amino acids, nucleic acids, sugars, and phospholipids are then recycled for energy generation and synthesis of new macromolecules to survive periods of starvation. Once such a catabolic machinery is in place it can be used for a multitude of other cellular processes, including degradation of pathogens, regulation of intercellular communication like inflammation and immune cell activation. In this review I will focus on its role in antigen presentation on major histocompatibility complex (MHC) molecules to $\mathrm{T}$ cells which utilizes proteolytic product display on MHC molecules at the cell surface.

\section{MACROAUTOPHAGY IN MHC CLASS II RESTRICTED ANTIGEN PRESENTATION}

The two classes of classical MHC molecules monitor different proteolytic machineries in cells by sampling a subset of these that they then transport to the cell surface for T cell stimulation (1416). Pathogen-derived or otherwise foreign peptides can then be recognized by the $\mathrm{T}$ cell repertoire that has been tolerized against self-peptides. MHC class I molecules present primarily products of proteasomal degradation that are then imported via the transporter associated with antigen presentation (TAP) into the endoplasmic reticulum and loaded in the MHC-I peptide-loading complex (17). After transport to the cell surface MHC class I molecules and their presented mostly nonameric peptides are then screened by cytotoxic $\mathrm{CD}^{+} \mathrm{T}$ cells. In contrast MHC class II molecules are loaded with usually longer peptides but a nonameric core sequence in MHC class II containing compartments (MIICs) which are late endosomes with lysosomal proteolytic capacity. MHC class II molecules are transported to MIICs as complexes with the invariant chain (Ii) that is then degraded and peptides loaded with the assistance of HLA-DM (H2-M in mice) onto MHC class II molecules (18). MHC class II molecules with their bound peptide ligands then migrate to the cell surface for surveillance by helper $\mathrm{CD} 4^{+} \mathrm{T}$ cells.

MHC class II restricted antigen presentation monitors therefore lysosomal proteolysis which degrades both endocytosed proteins and macroautophagy substrates (Figure 2). Indeed 20$30 \%$ of MHC class II ligands originate from cytosolic and nuclear sources, including the three ATG8 orthologues LC3B, GABARAP and GABARP-L2 $(19,20)$. MHC class II presentation of cytoplasmic constituents after macroautophagy was indeed 


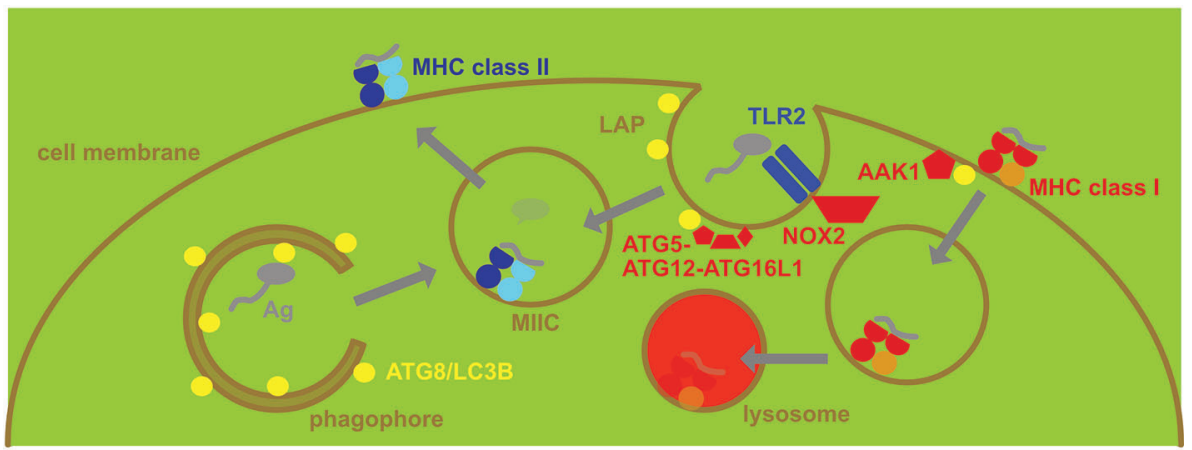

FIGURE 2 | Regulation of MHC presentation by the macroautophagy machinery. MHC class II gets loaded with peptides in MHC class II containing vesicles (MIICs). They receive antigens (Ag) from intracellular sources via macroautophagy and from extracellular sources via phagocytosis, including LC3-associated phagocytosis (LAP). LAP recruits ATG8/LC3B to the phagosomal membrane after for example TLR2 engagement and is dependent on NADPH oxidase 2 (NOX2). The ATG8/ LC3B lipidation complex of ATG5-ATG12-ATG16L1 conjugates ATG8/LC3B to the cytosolic side of phagosomes that then deliver the endocytosed antigen to MIICs In contrast MHC class I restricted antigen presentation is restricted by the macroautophagy machinery, supporting MHC class I internalization and lysosomal degradation. Adaptor associated kinase 1 (AAK1) is recruited to MHC class I molecules for the respective internalization.

initially demonstrated for three pathogen derived antigens, namely the nuclear antigen 1 of the Epstein Barr virus, bacterial neomycin phosphotransferase II (NeoR) and Ag85B of Mycobacterium tuberculosis (21-24). Processing of antigens for MHC class II presentation by macroautophagy can also be demonstrated by targeting them to phagophores via $\mathrm{N}$-terminal conjugation to LC3B (25). This increases intracellular antigen presentation on MHC class II, but not MHC class I molecules. Furthermore, LC3B can be found in MIICs $(25,26)$. Enhanced presentation of antigens on MHC class II molecules after macroautophagy targeting has now been demonstrated for viral and tumor proteins $(25,27-30)$. The above listed studies demonstrate that macroautophagy contributes to antigen processing for MHC class II presentation in a variety of cell types, including B cells $(19,21,22)$, epithelial cells (25), melanocytes (29) and myeloid antigen presenting cells (24, 28, 30). Therefore, MHC class II molecules can sample cytoplasmic antigens of pathogens for presentation via macroautophagy.

Possibly even more important, however, is the role of this pathway for $\mathrm{CD}^{+} \mathrm{T}$ cell tolerance induction. This tolerance induction requires in part MHC class II presentation by epithelial cells without significant phagocytic activity, such as thymic epithelial cells (TECs) (31). Medullary TECs (mTECs) express tissue-restricted self-antigens (TRAs) under the influence of the autoimmune regulator (AIRE). The intracellular expression of these TRAs leads to negative selection of autoimmune CD4 ${ }^{+}$ $\mathrm{T}$ cell clones in the thymus to ensure tolerance of the $\mathrm{T}$ cell repertoire against self. It was shown that macroautophagy deficient thymi were not able to sufficiently perform this negative selection, resulting in colitis and multi-organ inflammation in mice (32). Moreover, this primarily affected antigens that are endogenously expressed at low levels and cannot be efficiently transferred to neighboring cells for uptake, as is the case for most TRA expression in mTECs (33). As for the pathogen derived antigens, targeting of these to phagophores via $\mathrm{N}$-terminal conjugation to LC3B led to improved negative selection of $\mathrm{CD}^{+} \mathrm{T}$ cells. These findings suggest that efficient negative selection by TRAs in the thymus requires macroautophagy. Furthermore, regulatory $\mathrm{CD} 4^{+} \mathrm{T}$ cell stability in the periphery seems also to benefit from macroautophagy in dendritic cells (DCs) (34). However, this might not only depend on its role in endogenous self-antigen presentation on $\mathrm{MHC}$ class II molecules, but rather on a role of ATGs in co-stimulatory molecule expression. Nevertheless, macroautophagy can process cytoplasmic proteins for $\mathrm{MHC}$ class II presentation to $\mathrm{CD}^{+} \mathrm{T}$ cells and this seems particularly important for self-antigens that are expressed at low levels by cells with limited phagocytic potential in order to induce tolerance against these.

\section{LC3 ASSOCIATED PHAGOCYTOSIS IN MHC CLASS II RESTRICTED ANTIGEN PRESENTATION}

In addition it was, however, already noted in the first study that abolished ATG5 expression in DCs to study the influence of the macroautophagy machinery on MHC restricted antigen presentation and $\mathrm{T}$ cell responses in vivo that also extracellular antigen presentation to $\mathrm{CD} 4^{+} \mathrm{T}$ cells benefits from ATGs (35). Indeed, apart from the ATG1/ULK1 protein kinase complex the other components of the macroautophagy machinery also regulate phagocytosis. This was coined LC3-associated phagocytosis (LAP) (Figure 2), and the respective LC3B conjugation to the cytosolic side of phagosomes is primarily observed after co-engagement of pathogen associated molecular pattern receptors, such as toll-like receptor (TLR) 2, during uptake of extracellular material (36-40). During LAP, PI3P is deposited in a VPS34 dependent manner at the cytosolic side of phagosomes (41). This might be required to assemble efficiently NADPH oxidase (NOX2) at these phagosomes (42) and NOX2 mediated reactive oxygen species (ROS) production is required 
for $\operatorname{LAP}(37,41,43)$. The exact role of this ROS production is unclear to date, but the recruitment of the ATG8/LC3B lipidation seems to neither depend on ROS not PI3P at phagosomes (44). Instead it depends on the WD40 domain of ATG16L1 that is not required for macroautophagy $(44,45)$. The ATG16L1 dependent conjugation of LC3B to the cytosolic side of phagosomes is then removed prior to fusion with lysosomes as was observed by live cell imaging $(36,37)$. Dependent on the cell type LAP leads to accelerated fusion with lysosomes, delayed phagosome maturation or redirection of phagocytosed cargo to TLR containing endosomes $(36,37,46)$. This probably depends on the recruitment of different vesicular transport factors to LAP phagosomes in different cellular backgrounds.

Nevertheless, in both human and mouse phagocytes LAP supports MHC class II presentation of endocytosed antigens (37, $39,44,47,48)$. Since yeast cell wall components efficiently engage TLR2 Zymosan and Candida albicans spores or extracts were often used in these assays, and MHC class II presentation to Candida specific Th17 cells was monitored $(37,39)$. Phagocytosed Candida antigen presentation on MHC class II molecules to Th17 cells requires ATG5, ATG16L1 and more specifically the WD40 domain of ATG16L1 $(37,39,44)$. Bacterial outer membrane vesicles (OMVs) might also be processed via this LAP for regulatory $\mathrm{CD} 4^{+} \mathrm{T}$ cell stimulation (49). In addition to TLR mediated LAP induction it was also described that B cell stimulation activates ATG1/ULK1 independent use of the macroautophagy machinery (50). Accordingly, B cell receptor (BCR) mediated antigen uptake was found to use the ATG8/ LC3B lipidation machinery $(51,52)$. This allows BCR bound antigens to be delivered to TLR containing endosomes and to MIICs for antigen processing towards MHC class II presentation. Finally, myelin autoantigen presentation by DCs in the central nervous system (CNS) also depends on ATG5 and NOX2 as hallmarks of LAP, even so the receptor that mediates LC3B recruitment to phagocytosed oligodendrocyte derived apoptotic blebs has not been identified yet $(47,48)$. However, in mouse macrophages the phosphatidylserine binding scavenger receptor TIM4 was found to be involved in the clearance of apoptotic bodies by LAP (38). Thus, LAP supports autoimmune $\mathrm{CD} 4^{+} \mathrm{T}$ cell stimulation in the CNS for experimental autoimmune encephalomyelitis (EAE) development.

Therefore, ATG proteins support both intracellular and extracellular antigen presentation on MHC class II molecules to $\mathrm{CD}^{+} \mathrm{T}$ cells via macroautophagy and LAP, respectively.

\section{REGULATION OF MHC CLASS I RESTRICTED ANTIGEN PRESENTATION BY THE MACROAUTOPHAGY MACHINERY}

In contrast to the support of MHC class II restricted antigen presentation by the macroautophagy machinery, loss of ATGs leads to up-regulation of MHC class I restricted presentation of intracellular antigens (53-57). This affects both classical MHC class I molecules and the non-classical MHC class I molecule
CD1D that restricts glycolipid presentation to NKT cells. In these studies it was found that the stimulation of anti-viral, anti-tumor and alloreactive $\mathrm{CD}^{+} \mathrm{T}$ cell responses as well as anti-bacterial NKT cell immunity is enhanced in the absence of ATG3, ATG5, ATG7 or ATG16L1 (53-56). Higher classical and non-classical MHC class I expression on the surface of DCs and pancreatic carcinoma cells is at least in part responsible for this increased stimulation (54-56). This elevated surface expression of $\mathrm{MHC}$ class I molecules seems to be due to MHC class I targeting for lysosomal degradation by ATG proteins (Figure 2). In DCs this seems to be due to increased internalization and then degradation with the support of the macroautophagy machinery $(54,56)$. The identification of the adaptor associated kinase 1 (AAK1) and the adaptor complex AP2 point towards ATG proteins supporting clathrin mediated endocytosis $(54,56)$. This is reminiscent of the internalization of the amyloid precursor protein (APP) in Alzheimer's disease that was reported to require LC3 mediated recruitment of AP2 for efficient clathrin mediated turn-over $(58,59)$. In contrast in pancreatic carcinoma cells an NBR1 dependency of MHC class I degradation was observed and the authors suggested that macroautophagy of ER might redirect MHC class I molecules to lysosomes and therefore diminish surface expression for CD8 ${ }^{+}$ $\mathrm{T}$ cell stimulation (55). In both instances, deficiencies in the macroautophagy machinery, however, increase anti-viral (influenza and lymphocytic choriomeningitis virus) and antitumor $\mathrm{CD}^{+} \mathrm{T}$ cell responses via increased surface expression of MHC class I molecules. Lysosomal degradation of MHC class I molecules by the macroautophagy machinery seems to be also induced by ORF8 of the severe acute respiratory syndrome coronavirus 2 (SARS-CoV-2) (60). Moreover, autophagy inhibition can also redirect intracellular antigen degradation to proteasomes leading to increased MHC class I antigen presentation (57). Therefore, multiple pathways might account for increased MHC class I presentation of intracellular antigens by both somatic cells, such as tumor cells, and antigen presenting cells upon inhibition of the macroautophagy machinery.

With respect to extracellular antigen processing for MHC class I presentation, so called cross-presentation, the contribution of the macroautophagy machinery is not entirely clear yet. So far only soluble proteins have been described to benefit from the macroautophagy machinery in cross-presenting classical type 1 DCs and B cells $(61,62)$. However, long-term storage of antigen by DCs for cross-presentation was rather compromised by the macroautophagy machinery (63). Another function for antigen cross-presentation on MHC class I molecules was described for the macroautophagy machinery in antigen donor cells like virusinfected stromal or tumor cells $(64,65)$. In these studies, exocytosis of antigen containing vesicles that get efficiently cross-presented for $\mathrm{CD}^{+} \mathrm{T}$ cell stimulation by DCs seemed to benefit from the macroautophagy machinery. The packaging of antigens into the respective vesicles was improved by inhibiting both proteasomal and lysosomal degradation in the antigen donor cells (66-70). The respective pathway that utilizes the ATG8/ LC3B lipidation machinery for cross-presentable vesicle export could be overlapping or identical to LC3-dependent extracellular vesicle loading and secretion (LDELS) (71-76). Potentially more 
than one exocytosis pathway seems to benefit from the macroautophagy machinery and neutral sphingomyelinase 2 (nSMase2) or Golgi reassembly stacking proteins (GRASPs) have been reported to be involved in this ATG supported exocytosis. Thus, extracellular antigen processing for MHC class I presentation seems to benefit from a functional macroautophagy machinery in both antigen donor cells and for short-term crosspresentation of certain antigen formulations also in antigen presenting cells, while the autophagic machinery limits MHC class I surface expression for intracellular antigen presentation.

\section{CONCLUSIONS AND FUTURE PERSPECTIVES}

Lysosomal and proteasomal protein degradation are the two main proteolytic machineries of cells and their products sampled by MHC class II and I molecules for $\mathrm{CD}^{+}$and $\mathrm{CD}^{+} \mathrm{T}$ cell stimulation, respectively. Accordingly, macroautophagy as a component of lysosomal degradation, targeting cytoplasmic constituents, supports $\mathrm{MHC}$ class II restricted antigen presentation to helper $\mathrm{CD}^{+} \mathrm{T}$ cells. Recent years have, however, demonstrated that the same molecular machinery that supports macroautophagy also regulates endocytosis and exocytosis. While the role of ATG proteins in endocytosis seems to promote $\mathrm{MHC}$ class II presentation of phagocytosed antigens via LAP and inhibit MHC class I presentation of intracellular antigens through lysosomal degradation of MHC class I plus peptide complexes, exocytosis might package antigens optimally for processing onto MHC class I molecules during cross-

\section{REFERENCES}

1. Tsukada M, Ohsumi Y. Isolation and characterization of autophagy-defective mutants of Saccharomyces cerevisiae. FEBS Lett (1993) 333:169-74. doi: 10.1016/0014-5793(93)80398-E

2. Mizushima N, Yoshimori T, Ohsumi Y. The Role of Atg Proteins in Autophagosome Formation. Annu Rev Cell Dev Biol (2011) 27:107-32. doi: 10.1146/annurev-cellbio-092910-154005

3. Hu Z, Raucci S, Jaquenoud M, Hatakeyama R, Stumpe M, Rohr R, et al. Multilayered Control of Protein Turnover by TORC1 and Atg1. Cell Rep (2019) 28:3486-96.e6. doi: 10.1016/j.celrep.2019.08.069

4. Nakatogawa H, Ichimura Y, Ohsumi Y. Atg8, a ubiquitin-like protein required for autophagosome formation, mediates membrane tethering and hemifusion. Cell (2007) 130:165-78. doi: 10.1016/j.cell.2007.05.021

5. Osawa T, Kotani T, Kawaoka T, Hirata E, Suzuki K, Nakatogawa H, et al. Atg2 mediates direct lipid transfer between membranes for autophagosome formation. Nat Struct Mol Biol (2019) 26:281-8. doi: 10.1038/s41594-0190203-4

6. Matoba K, Kotani T, Tsutsumi A, Tsuji T, Mori T, Noshiro D, et al. Atg9 is a lipid scramblase that mediates autophagosomal membrane expansion. Nat Struct Mol Biol (2020) 27:1185-93. doi: 10.1038/s41594-020-00538-6

7. Maruyama T, Noda NN. Autophagy-regulating protease Atg4: structure, function, regulation and inhibition. J Antibiot (Tokyo) (2017) 71:72-8. doi: 10.1038/ja.2017.104

8. Rogov V, Dotsch V, Johansen T, Kirkin V. Interactions between autophagy receptors and ubiquitin-like proteins form the molecular basis for selective autophagy. Mol Cell (2014) 53:167-78. doi: 10.1016/j.molcel.2013.12.014

9. Verlhac P, Gregoire IP, Azocar O, Petkova DS, Baguet J, Viret C, et al. Autophagy receptor NDP52 regulates pathogen-containing autophagosome presentation. Since not all modules of the macroautophagy machinery are used in all these pathways, regulation of specific ATG proteins in antigen donor or presenting cells might be used to influence MHC class I or II presentation in the tumor microenvironment or during viral infections. However, which manipulation might be beneficial in which setting needs to be determined on a case by case basis, considering, in addition to the role of the macroautophagy machinery for $\mathrm{MHC}$ restricted antigen presentation, also its anti-inflammatory role in myeloid cells and its pro-survival role in lymphocytes as well as in virus infected and tumor cells.

\section{AUTHOR CONTRIBUTIONS}

The author confirms being the sole contributor of this work and has approved it for publication.

\section{FUNDING}

My research is supported by Cancer Research Switzerland (KFS-4091-02-2017 and KFS-4962-02-2020), KFSP-Precision ${ }^{\mathrm{MS}}$ and HMZ ImmunoTargET of the University of Zurich, the Cancer Research Center Zurich, the Vontobel Foundation, the Baugarten Foundation, the Sobek Foundation, the Swiss Vaccine Research Institute, Roche, Novartis and the Swiss National Science Foundation (310030B_182827, 310030L_197952/1, and CRSII5_180323).

maturation. Cell Host Microbe (2015) 17:515-25. doi: 10.1016/ j.chom.2015.02.008

10. McEwan DG, Popovic D, Gubas A, Terawaki S, Suzuki H, Stadel D, et al. PLEKHM1 Regulates Autophagosome-Lysosome Fusion through HOPS Complex and LC3/GABARAP Proteins. Mol Cell (2015) 57:39-54. doi: 10.1016/j.molcel.2014.11.006

11. Itakura E, Kishi-Itakura C, Mizushima N. The hairpin-type tail-anchored SNARE syntaxin 17 targets to autophagosomes for fusion with endosomes/ lysosomes. Cell (2012) 151:1256-69. doi: 10.1016/j.cell.2012.11.001

12. Matsui T, Jiang P, Nakano S, Sakamaki Y, Yamamoto H, Mizushima N. Autophagosomal YKT6 is required for fusion with lysosomes independently of syntaxin 17. J Cell Biol (2018) 217:2633-45. doi: 10.1083/jcb.201712058

13. Settembre C, Di Malta C, Polito VA, Garcia Arencibia M, Vetrini F, Erdin S, et al. TFEB links autophagy to lysosomal biogenesis. Science (2011) 332:142933. doi: $10.1126 /$ science. 1204592

14. Trombetta ES, Mellman I. Cell biology of antigen processing in vitro and in vivo. Annu Rev Immunol (2005) 23:975-1028. doi: 10.1146/annurev. immunol.22.012703.104538

15. Dersh D, Holly J, Yewdell JW. A few good peptides: MHC class I-based cancer immunosurveillance and immunoevasion. Nat Rev Immunol (2020) 20:644. doi: 10.1038/s41577-020-0390-6

16. Moore TV, Nishimura MI. Improved MHC II epitope prediction - a step towards personalized medicine. Nat Rev Clin Oncol (2020) 17:71-2. doi: 10.1038/s41571-019-0315-0

17. Blees A, Januliene D, Hofmann T, Koller N, Schmidt C, Trowitzsch S, et al. Structure of the human MHC-I peptide-loading complex. Nature (2017) 551:525-8. doi: 10.1038/nature24627

18. Pos W, Sethi DK, Call MJ, Schulze MS, Anders AK, Pyrdol J, et al. Crystal structure of the HLA-DM-HLA-DR1 complex defines mechanisms for rapid peptide selection. Cell (2012) 151:1557-68. doi: 10.1016/j.cell.2012.11.025 
19. Dengjel J, Schoor O, Fischer R, Reich M, Kraus M, Muller M, et al. Autophagy promotes MHC class II presentation of peptides from intracellular source proteins. Proc Natl Acad Sci USA (2005) 102:7922-7. doi: 10.1073/pnas. 0501190102

20. Suri A, Walters JJ, Rohrs HW, Gross ML, Unanue ER. First Signature of Islet \{beta\}-Cell-Derived Naturally Processed Peptides Selected by Diabetogenic Class II MHC Molecules. J Immunol (2008) 180:3849-56. doi: 10.4049/ jimmunol.180.6.3849

21. Paludan C, Schmid D, Landthaler M, Vockerodt M, Kube D, Tuschl T, et al. Endogenous MHC class II processing of a viral nuclear antigen after autophagy. Science (2005) 307:593-6. doi: 10.1126/science.1104904

22. Leung CS, Haigh TA, Mackay LK, Rickinson AB, Taylor GS. Nuclear location of an endogenously expressed antigen, EBNA1, restricts access to macroautophagy and the range of CD4 epitope display. Proc Natl Acad Sci U S A (2010) 107:2165-70. doi: 10.1073/pnas.0909448107

23. Nimmerjahn F, Milosevic S, Behrends U, Jaffee EM, Pardoll DM, Bornkamm GW, et al. Major histocompatibility complex class II-restricted presentation of a cytosolic antigen by autophagy. Eur J Immunol (2003) 33:1250-9. doi: 10.1002/eji.200323730

24. Jagannath C, Lindsey DR, Dhandayuthapani S, Xu Y, Hunter RLJr, Eissa NT. Autophagy enhances the efficacy of BCG vaccine by increasing peptide presentation in mouse dendritic cells. Nat Med (2009) 15:267-76. doi: $10.1038 / \mathrm{nm} .1928$

25. Schmid D, Pypaert M, Münz C. MHC class II antigen loading compartments continuously receive input from autophagosomes. Immunity (2007) 26:79-92. doi: 10.1016/j.immuni.2006.10.018

26. Kasai M, Tanida I, Ueno T, Kominami E, Seki S, Ikeda T, et al. Autophagic compartments gain access to the MHC class II compartments in thymic epithelium. J Immunol (2009) 183:7278-85. doi: 10.4049/jimmunol.0804087

27. Comber JD, Robinson TM, Siciliano NA, Snook AE, Eisenlohr LC. Functional Macroautophagy Induction by Influenza A Virus Without a Contribution to MHC-Class II Restricted Presentation. J Virol (2011) 85:6453-63. doi: 10.1128/JVI.02122-10

28. Jin Y, Sun C, Feng L, Li P, Xiao L, Ren Y, et al. Regulation of SIV antigenspecific $\mathrm{CD}^{+} \mathrm{T}$ cellular immunity via autophagosome-mediated MHC II molecule-targeting antigen presentation in mice. PloS One (2014) 9:e93143. doi: 10.1371/journal.pone.0093143

29. Fonteneau JF, Brilot F, Münz C, Gannage M. The Tumor Antigen NY-ESO-1 Mediates Direct Recognition of Melanoma Cells by $\mathrm{CD} 4^{+} \mathrm{T}$ Cells after Intercellular Antigen Transfer. J Immunol (2016) 196:64-71. doi: 10.4049/ jimmunol.1402664

30. Coulon PG, Richetta C, Rouers A, Blanchet FP, Urrutia A, Guerbois M, et al. HIV-Infected Dendritic Cells Present Endogenous MHC Class II-Restricted Antigens to HIV-Specific CD4 ${ }^{+}$T Cells. J Immunol (2016) 197:517-32. doi: 10.4049/jimmunol.1600286

31. Kyewski B, Klein L. A central role for central tolerance. Annu Rev Immunol (2006) 24:571-606. doi: 10.1146/annurev.immunol.23.021704.115601

32. Nedjic J, Aichinger M, Emmerich J, Mizushima N, Klein L. Autophagy in thymic epithelium shapes the T-cell repertoire and is essential for tolerance. Nature (2008) 455:396-400. doi: 10.1038/nature07208

33. Aichinger M, Wu C, Nedjic J, Klein L. Macroautophagy substrates are loaded onto MHC class II of medullary thymic epithelial cells for central tolerance. J Exp Med (2013) 210:287-300. doi: 10.1084/jem.20122149

34. Niven J, Madelon N, Page N, Caruso A, Harle G, Lemeille S, et al. Macroautophagy in Dendritic Cells Controls the Homeostasis and Stability of Regulatory T Cells. Cell Rep (2019) 28:21-9.e6. doi: 10.1016/j.celrep.2019.05.110

35. Lee HK, Mattei LM, Steinberg BE, Alberts P, Lee YH, Chervonsky A, et al. In vivo requirement for Atg5 in antigen presentation by dendritic cells. Immunity (2010) 32:227-39. doi: 10.1016/j.immuni.2009.12.006

36. Sanjuan MA, Dillon CP, Tait SW, Moshiach S, Dorsey F, Connell S, et al. Tolllike receptor signalling in macrophages links the autophagy pathway to phagocytosis. Nature (2007) 450:1253-7. doi: 10.1038/nature06421

37. Romao S, Gasser N, Becker AC, Guhl B, Bagajic M, Vanoaica LD, et al. Essential autophagy proteins stabilize pathogen containing phagosomes for prolonged MHC class II antigen processing. J Cell Biol (2013) 203:757-66. doi: $10.1083 /$ jcb. 201308173

38. Martinez J, Almendinger J, Oberst A, Ness R, Dillon CP, Fitzgerald P, et al. Microtubule-associated protein 1 light chain 3 alpha (LC3)-associated phagocytosis is required for the efficient clearance of dead cells. Proc Natl Acad Sci USA (2011) 108:17396-401. doi: 10.1073/pnas.1113421108

39. Ma J, Becker C, Lowell CA, Underhill DM. Dectin-1-triggered Recruitment of Light Chain 3 Protein to Phagosomes Facilitates Major Histocompatibility Complex Class II Presentation of Fungal-derived Antigens. J Biol Chem (2012) 287:34149-56. doi: 10.1074/jbc.M112.382812

40. Florey O, Kim SE, Sandoval CP, Haynes CM, Overholtzer M. Autophagy machinery mediates macroendocytic processing and entotic cell death by targeting single membranes. Nat Cell Biol (2011) 13:1335-43. doi: 10.1038/ ncb2363

41. Martinez J, Malireddi RK, Lu Q, Cunha LD, Pelletier S, Gingras S, et al. Molecular characterization of LC3-associated phagocytosis reveals distinct roles for Rubicon, NOX2 and autophagy proteins. Nat Cell Biol (2015) 17:893-906. doi: $10.1038 / \mathrm{ncb} 3192$

42. Ellson C, Davidson K, Anderson K, Stephens LR, Hawkins PT. PtdIns3P binding to the PX domain of p40phox is a physiological signal in NADPH oxidase activation. EMBO J (2006) 25:4468-78. doi: 10.1038/sj.emboj.7601346

43. Huang J, Canadien V, Lam GY, Steinberg BE, Dinauer MC, Magalhaes MA, et al. Activation of antibacterial autophagy by NADPH oxidases. Proc Natl Acad Sci U S A (2009) 106:6226-31. doi: 10.1073/pnas.0811045106

44. Fletcher K, Ulferts R, Jacquin E, Veith T, Gammoh N, Arasteh JM, et al. The WD40 domain of ATG16L1 is required for its non-canonical role in lipidation of LC3 at single membranes. EMBO J (2018) 37:e97840. doi: 10.15252/ embj. 201797840

45. Rai S, Arasteh M, Jefferson M, Pearson T, Wang Y, Zhang W, et al. The ATG5binding and coiled coil domains of ATG16L1 maintain autophagy and tissue homeostasis in mice independently of the WD domain required for LC3associated phagocytosis. Autophagy (2019) 15:599-612. doi: 10.1080/ 15548627.2018.1534507

46. Henault J, Martinez J, Riggs JM, Tian J, Mehta P, Clarke L, et al. Noncanonical autophagy is required for type I interferon secretion in response to DNA-immune complexes. Immunity (2012) 37:986-97. doi: 10.1016/j.immuni.2012.09.014

47. Keller CW, Sina C, Kotur MB, Ramelli G, Mundt S, Quast I, et al. ATGdependent phagocytosis in dendritic cells drives myelin-specific $\mathrm{CD} 4^{+} \mathrm{T}$ cell pathogenicity during CNS inflammation. Proc Natl Acad Sci USA (2017) 114: E11228-37. doi: 10.1073/pnas.1713664114

48. Keller CW, Kotur MB, Mundt S, Dokalis N, Ligeon LA, Shah AM, et al. CYBB/ NOX2 in conventional DCs controls T cell encephalitogenicity during neuroinflammation. Autophagy (2020). doi: 10.1080/15548627.2020.1756678

49. Chu H, Khosravi A, Kusumawardhani IP, Kwon AH, Vasconcelos AC, Cunha $\mathrm{LD}$, et al. Gene-microbiota interactions contribute to the pathogenesis of inflammatory bowel disease. Science (2016) 352:1116-20. doi: 10.1126/ science.aad 9948

50. Martinez-Martin N, Maldonado P, Gasparrini F, Frederico B, Aggarwal S, Gaya M, et al. A switch from canonical to noncanonical autophagy shapes B cell responses. Science (2017) 355:641-7. doi: 10.1126/science.aal3908

51. Arbogast F, Arnold J, Hammann P, Kuhn L, Chicher J, Murera D, et al. ATG5 is required for B cell polarization and presentation of particulate antigens. Autophagy (2019) 15:280-94. doi: 10.1080/15548627.2018.1516327

52. Chaturvedi A, Dorward D, Pierce SK. The B cell receptor governs the subcellular location of Toll-like receptor 9 leading to hyperresponses to DNA-containing antigens. Immunity (2008) 28:799-809. doi: 10.1016/ j.immuni.2008.03.019

53. Hubbard-Lucey VM, Shono Y, Maurer K, West ML, Singer NV, Ziegler CGK, et al. Autophagy Gene Atg1611 Prevents Lethal T Cell Alloreactivity Mediated by Dendritic Cells. Immunity (2014) 41:579-91. doi: 10.1016/ j.immuni.2014.09.011

54. Loi M, Muller A, Steinbach K, Niven J, Barreira da Silva R, Paul P, et al. Macroautophagy Proteins Control MHC Class I Levels on Dendritic Cells and Shape Anti-viral CD8 ${ }^{+}$T Cell Responses. Cell Rep (2016) 15:1076-87. doi: 10.1016/j.celrep.2016.04.002

55. Yamamoto K, Venida A, Yano J, Biancur DE, Kakiuchi M, Gupta S, et al. Autophagy promotes immune evasion of pancreatic cancer by degrading MHC-I. Nature (2020) 581:100-5. doi: 10.1038/s41586-020-2229-5

56. Keller CW, Loi M, Ewert S, Quast I, Theiler R, Gannage M, et al. The autophagy machinery restrains iNKT cell activation through CD1D1 internalization. Autophagy (2017) 13:1025-36. doi: 10.1080/15548627.2017. 1297907 
57. Wenger T, Terawaki S, Camosseto V, Abdelrassoul R, Mies A, Catalan N, et al. Autophagy inhibition promotes defective neosynthesized proteins storage in ALIS, and induces redirection toward proteasome processing and MHCIrestricted presentation. Autophagy (2012) 8:350-63. doi: 10.4161/auto.18806

58. Tian Y, Bustos V, Flajolet M, Greengard P. A small-molecule enhancer of autophagy decreases levels of Abeta and APP-CTF via Atg5-dependent autophagy pathway. FASEB J (2011) 25:1934-42. doi: 10.1096/fj.10-175158

59. Tian Y, Chang JC, Fan EY, Flajolet M, Greengard P. Adaptor complex AP2/ PICALM, through interaction with LC3, targets Alzheimer's APP-CTF for terminal degradation via autophagy. Proc Natl Acad Sci USA (2013) 110:17071-6. doi: 10.1073/pnas.1315110110

60. Zhang Y, Zhang J, Chen Y, Luo B, Yuan Y, Huang F, et al. The ORF8 Protein of SARS-CoV-2 Mediates Immune Evasion through Potently Downregulating MHC-I. bioRxiv (2020). doi: 10.1101/2020.05.24.111823

61. Mintern JD, Macri C, Chin WJ, Panozza SE, Segura E, Patterson NL, et al. Differential use of autophagy by primary dendritic cells specialized in crosspresentation. Autophagy (2015) 11:906-17. doi: 10.1080/15548627.2015. 1045178

62. Dasari V, Rehan S, Tey SK, Smyth MJ, Smith C, Khanna R. Autophagy and proteasome interconnect to coordinate cross-presentation through MHC class I pathway in B cells. Immunol Cell Biol (2016) 94:964-74. doi: 10.1038/icb.2016.59

63. Ho NI, Camps MGM, Verdoes M, Münz C, Ossendorp F. Autophagy regulates long-term cross-presentation by murine dendritic cells. Eur J Immunol (2020). doi: 10.1002/eji.202048961

64. Uhl M, Kepp O, Jusforgues-Saklani H, Vicencio JM, Kroemer G, Albert ML. Autophagy within the antigen donor cell facilitates efficient antigen crosspriming of virus-specific CD8 ${ }^{+}$T cells. Cell Death Differ (2009) 16:991-1005. doi: $10.1038 / \mathrm{cdd} .2009 .8$

65. Li Y, Wang LX, Yang G, Hao F, Urba WJ, Hu HM. Efficient cross-presentation depends on autophagy in tumor cells. Cancer Res (2008) 68:6889-95. doi: 10.1158/0008-5472.CAN-08-0161

66. Li Y, Hahn T, Garrison K, Cui ZH, Thorburn A, Thorburn J, et al. The vitamin E analogue alpha-TEA stimulates tumor autophagy and enhances antigen cross-presentation. Cancer Res (2012) 72:3535-45. doi: 10.1158/00085472.CAN-11-3103

67. Li Y, Wang LX, Pang P, Cui Z, Aung S, Haley D, et al. Tumor-derived autophagosome vaccine: mechanism of cross-presentation and therapeutic efficacy. Clin Cancer Res (2011) 17:7047-57. doi: 10.1158/1078-0432.CCR-110951

68. Fan J, Wu Y, Jiang M, Wang L, Yin D, Zhang Y, et al. IFN-DC Loaded with Autophagosomes containing Virus Antigen is Highly Efficient in Inducing
Virus-Specific Human T Cells. Int J Med Sci (2019) 16:741-50. doi: 10.7150/ ijms. 31830

69. Ye W, Xing Y, Paustian C, van de Ven R, Moudgil T, Hilton TL, et al. Crosspresentation of viral antigens in dribbles leads to efficient activation of virusspecific human memory T cells. J Transl Med (2014) 12:100. doi: 10.1186/ 1479-5876-12-100

70. Yi Y, Zhou Z, Shu S, Fang Y, Twitty C, Hilton TL, et al. Autophagy-assisted antigen cross-presentation: Autophagosome as the argo of shared tumorspecific antigens and DAMPs. Oncoimmunology (2012) 1:976-8. doi: 10.4161/ onci.20059

71. Leidal AM, Huang HH, Marsh T, Solvik T, Zhang D, Ye J, et al. The LC3conjugation machinery specifies the loading of RNA-binding proteins into extracellular vesicles. Nat Cell Biol (2020) 22:187-99. doi: 10.1038/s41556019-0450-y

72. Duran JM, Anjard C, Stefan C, Loomis WF, Malhotra V. Unconventional secretion of Acb1 is mediated by autophagosomes. J Cell Biol (2010) 188:52736. doi: $10.1083 /$ jcb. 200911154

73. Manjithaya R, Anjard C, Loomis WF, Subramani S. Unconventional secretion of Pichia pastoris Acb1 is dependent on GRASP protein, peroxisomal functions, and autophagosome formation. J Cell Biol (2010) 188:537-46. doi: $10.1083 /$ jcb.200911149

74. Dupont N, Jiang S, Pilli M, Ornatowski W, Bhattacharya D, Deretic V. Autophagy-based unconventional secretory pathway for extracellular delivery of IL-1beta. EMBO J (2011) 30:4701-11. doi: 10.1038/emboj.2011.398

75. Kimura T, Jia J, Kumar S, Choi SW, Gu Y, Mudd M, et al. Dedicated SNAREs and specialized TRIM cargo receptors mediate secretory autophagy. EMBO J (2017) 36:42-60. doi: 10.15252/embj.201695081

76. Zhang M, Kenny S, Ge L, Xu K, Schekman R. Translocation of interleukin1 beta into a vesicle intermediate in autophagy-mediated secretion. Elife (2015) 4:e11205. doi: 10.7554/eLife.11205

Conflict of Interest: The author declares that the research was conducted in the absence of any commercial or financial relationships that could be construed as a potential conflict of interest.

Copyright (C) 2021 Münz. This is an open-access article distributed under the terms of the Creative Commons Attribution License (CC BY). The use, distribution or reproduction in other forums is permitted, provided the original author(s) and the copyright owner(s) are credited and that the original publication in this journal is cited, in accordance with accepted academic practice. No use, distribution or reproduction is permitted which does not comply with these terms. 